\title{
PROGRAMA DE INTERVENCIÓN EDUCATIVA PARA EL TRATAMIENTO DE LA OBESIDAD INFANTIL BASADO EN VIDEOJUEGOS ACTIVOS
}

\author{
Carina Soledad González González (Universidad de La Laguna)* \\ Nazaret Gómez del Rio (Universidad de La Laguna)** \\ Vicente Navarro Adelantado (Universidad de La Laguna)***
}

\section{RESUMEN}

La obesidad infantil es la enfermedad crónica con mayor prevalencia en los países desarrollados y en vías de desarrollo. Los últimos estudios mostraron que Canarias es la comunidad española con el índice de obesidad infantil más alto. Sin embargo, esta enfermedad es fácil de prevenir, educando desde niños en hábitos saludables, y el desarrollo de las TICs en general y los videojuegos en particular, representan una oportunidad de trabajar para alcanzar un cambio. Por ello, en este trabajo se presenta el diseño de un programa de intervención educativa para el tratamiento de la obesidad infantil. Este programa está destinado a niños y niñas de 8 a 12 años de edad con obesidad y que acuden por primera vez al Servicio de Pediatría del Hospital Universitario de Canarias, y actualmente está siendo validado. Los resultados parciales muestran que la satisfacción de los menores con el programa es alta, destacando el enfoque en juegos. Asimismo, el 100\% de los niños y niñas creen que han aprendido a través del programa de intervención educativa, y que tanto la actividad física $(91,7 \%)$, como la alimentación saludable $(90,9 \%)$ han cambiado sus hábitos de vida.

Palabras clave: Obesidad-infantil. Tratamiento. Educación. Hábitos saludables. Videojuegos.

\section{ABSTRACT \\ EDUCATIONAL INTERVENTION PROGRAM FOR THE TREATMENT OF CHILDHOOD OBESITY BASED ON ACTIVE VIDEO GAMES \\ Childhood obesity is the most prevalent chronic disease, both in developed and in developing countries. Recent studies showed that the Canaries is the Spanish community with the highest rate of childhood obesity. However, this disease is easily preventable by educating children from unhealthy habits, and the development of ICT in general, and video games in particular, represent an opportunity to work towards}

* Doctora en Informática. Grupo de Investigación Interacción, Tecnología y Educación (ITED). Escuela de Ingeniería y Tecnología. Universidad de La Laguna. La Laguna. Tenerife. 38204. España. E-mail: cjgonza@ull.edu.es

** Mg. Investigación, Gestión y Calidad en Cuidados para la Salud. Grupo de Investigación Interacción, Tecnología y Educación (ITED). Facultad de Ciencias de La Salud. La Laguna. . Universidad de La Laguna. Tenerife. 38204. España. E-mail: ngomezde@ull.edu.es

*** Doctor en Educación Física. Grupo de Investigación Interacción, Tecnología y Educación (ITED). Facultad de Educación. Universidad de La Laguna. La Laguna. Tenerife. 38204. España. E-mail: ngomezde@ull.edu.es 
the change. So, in this paper we present the design of an educational intervention program for treatment of childhood obesity. This program is intended for children from 8 to 12 years old with obesity attending for the first time at the Pediatric Service of the Hospital Universitario de Canarias. This program is currently being validated. Partial results show that satisfaction under the program is high, highlighting the focus on games. Also, $100 \%$ of children believe they have learned through educational intervention program, and both physical activity (91.7\%), as healthy eating (90.9\%) have changed their lifetime habits.

Keywords: Childhood obesity. Treatment. Education. Healthy habits. Video games.

\section{RESUMO}

\section{PROGRAMA DE INTERVENÇÃO EDUCATIVA PARA O TRATAMENTO DA OBESIDADE INFANTIL BASEADO EM VÍDEOJOGOS ATIVOS}

A obesidade infantil é a doença crônica mais prevalente nos países desenvueltos e em desenvolvimento. Estudos recentes mostraram que as Canárias é a comunidade espanhola com a maior taxa de obesidade infantil. No entanto, esta doença é facilmente evitável se educar as crianças com hábitos saudáveis. E o desenvolvimento das TICs em geral e dos videojogos em particular representam uma oportunidade de trabalhar para uma mudança. Por isso, neste trabalho se apresenta a concepção de um programa de intervenção educativa para o tratamento da obesidade infantil. Este programa é destinado a crianças de 8 a 12 anos com obesidade e que vão pela primeira vez ao Serviço de Pediatria do Hospital Universitário de Canárias, e atualmente está sendo validado. Os resultados parciais mostram que a satisfação no âmbito do programa é alta, com destaque para o foco em jogos. Além disso, 100\% das crianças acreditam que aprenderam através do programa de intervenção educativa, e que tanto a atividade física (91,7\%), como uma alimentação saudável (90,9\%) mudaram seus hábitos de vida.

Palavras-chave: Obesidade-infantil. Tratamento. Educação. Hábitos saudáveis Videogames.

\section{Introducción}

En 2004, la OMS declara la obesidad como la epidemia del s. XXI tras alcanzar proporciones mundiales, aunque ya desde 1998 en su informe mundial de la salud hablaba de la obesidad como un problema de salud emergente (ORGANIZACIÓN MUNDIAL DE LA SALUD, 2004, 2014). Esta epidemia afecta principalmente a los países desarrollados y en vías de desarrollo, aunque ya no es exclusivo de países de altos ingresos y empieza a estar presente en países pobres, afectando a toda la población desde la infancia hasta la edad adulta. En España, esta enfermedad la padece el 62\% de su población según datos del Estudio de Nutrición y Riesgo Cardiovascular en España (SOCIEDAD
ESPAÑOLA PARA EL ESTUDIO DE LA OBESIDAD, 2013). El sobrepeso y la obesidad son el quinto factor de riesgo de defunción en el mundo, y se le atribuyen cerca de tres millones de muertes al año (FRENK, 2013). Los problemas de salud derivados de padecer sobrepeso u obesidad se calculan en el 58\% de la carga de diabetes, el $21 \%$ de cardiopatías isquémicas, y entre el $8 \%$ y $42 \%$ de algunos cánceres y estos riesgos crecen en proporción al aumento de peso corporal (ORGANIZACIÓN MUNDIAL DE LA SALUD, 2014). La obesidad ha ido aumentando de forma progresiva en las últimas décadas (AGENCIA ESPAÑOLA DE SEGURIDAD ALIMENTARIA, 2005; EZZATI; RIBOLI, 
2013), y la obesidad en la población pediátrica se ha convertido en uno de los problemas más graves de la salud pública, estimándose que cerca de 42 millones de niños menores de cinco años tenían sobrepeso u obesidad en 2010, 35 de los cuales viven en países desarrollados. 1

Los responsables del crecimiento de las cifras de obesidad infantil en el mundo son los cambios en los estilos de vida, y el desarrollo social y laboral que han sufrido la mayoría de las poblaciones: los alimentos tradicionales han sido reemplazados por alimentos más ricos en calorías, grasas, y azúcares, además de favorecerse una mala alimentación basada en bollería (industria alimentaria) aperitivos, sal y pobre en fruta, verdura, hortalizas y legumbres, se realiza poca o ninguna actividad física debido, entre otras razones, al mayor nivel de urbanización y mecanización, a la evolución de los sistemas de transporte, los cambios en políticas sociales y sanitarias, el planteamiento urbano que deja poco espacio a zona verdes, y al largo tiempo que pasan los escolares realizando actividades de ocio sedentarias frente al ordenador o al televisor son algunos de ellos (CUSSÓ SEGURA; GARRABOU SEGURA, 2007).

En España, los cambios de estilo de vida y al desarrollo del Sistema Nacional Sanitario, entre otros factores, provocaron la aparición de nuevos problemas sanitarios, las enfermedades no transmisibles, como la obesidad sustituyeron a las enfermedades infecciosas. En Canarias este crecimiento de las cifras de obesidad se debe al desarrollo económico que se produjo con el turismo y a la migración del campo a las grandes ciudades, lo que conllevo un cambio en los hábitos alimenticios, y la sustitución de la comida tradicional por una rica en grasas (SOCIEDAD ESPAÑOLA PARA EL ESTUDIO DE LA OBESIDAD, 2013). En Brasil, en las últimas tres décadas el número total de niños y niñas brasileños con sobrepeso aumentó de alrededor del 9\% en los años 70 a aproximadamente el $13 \%$ en los años 80 , llegando a un $33,5 \%$ en 2009 , cuando se realizó la última encuesta sobre el tema la Encuesta

1 Este trabajo ha sido posible gracias al Proyecto "Programa de Videojuegos activos para el Tratamiento Ambulatorio de la Obesidad (PROVITAO)" Ref. OBE05, financiado por la Fundación Cajacanarias dentro de la línea de Proyectos de Investigación Sanitaria. de Presupuestos Familiares (EPF) del Instituto Brasileño de Geografía y Estadística (IBGE) (INSTITUTO BRASILEIRO DE DEFESA DO CONSUMIDOR, 2015). Una de las principales razones de este aumento es el cambio de hábitos en la alimentación de los brasileños que han cambiado de consumir productos caseros a productos ultra procesados (INSTITUTO BRASILEIRO DE DEFESA DO CONSUMIDOR, 2015).

En este artículo veremos algunas investigaciones y programas de intervención que han sido desarrollados para tratar y prevenir la obesidad infantil, y en particular, nos centraremos en aquellos estudios que utilizan videojuegos y miden su eficacia. Luego, presentaremos el diseño de un programa de intervención educativo utilizando videojuegos activos y juegos motores para el apoyo al tratamiento ambulatorio de la obesidad (PROGRAMA DE VIDEOJUEGOS ACTIVOS PARA EL TRATAMIENTO AMBULATORIO DE LA OBESIDAD, 2016), diseñado y desarrollado por el Grupo de Investigación Interacción, Tecnología y Educación (ITED) de la Universidad de La Laguna, $\mathrm{y}$ algunos resultado parciales del mismo.

\section{Videojuegos y promoción de hábitos de vida saludables}

En la actual sociedad del s. XIX, rodeada de tecnología y con elevados índices de sedentarismo, los videojuegos activos se presentan como una nueva herramienta que puede ayudar a la sociedad no solo en su ámbito de entretenimiento sino también puede reportar beneficios para la salud pública (ALVES, 2013). Algunos estudios recientes destacan el papel que pueden desempeñar los videojuegos en la promoción de conductas saludables así como las posibilidades que ofrecen para hacer frente a la obesidad infantil, ya hacen referencia al potencial de los videojuegos activos como herramienta de promoción de la actividad física (BARANOWSKI et al, 2008; BELTRÁN-CARRILLO; VALENCIA-PERIS; MOLINA-ALVENTOS, 2011; HILLIER, 2008).

Teniendo presente esta realidad, se hace necesario encontrar nuevas maneras de motivar a los menores en la práctica de la actividad física. Los videojuegos activos pueden ser útiles para diseñar 
programas de ejercicio personalizados y conseguir aumentar la actividad entre niños y adolescentes con sobrepeso. Actualmente, se ha comprobado que los escenarios virtuales reducen la sensación de esfuerzo del niño además de aumentar su motivación, lo que convierte a los videojuegos activos en una nueva alternativa para el mantenimiento de un estilo de vida activa (LÓPEZ et al, 2015; ZURANO CONCHES, 2011). Por lo tanto, se hace evidente que los videojuegos activos pueden convertirse en la nueva herramienta para entender la relación entre los videojuegos y la salud de la población infanto-juvenil.
El término Exergaming deriva de la combinación de las palabras exercise y gaming, poniendo en común el juego virtual y el ejercicio (CUBEROS et al, 2015), y aunque esta definición se ha usado durante mucho tiempo para referirse a los videojuegos activos, actualmente se entiende que existen diversas categorías en este ámbito y que este término se refiere sólo a un tipo de juegos activos. Por lo tanto, podemos clasificar los videojuegos en tres tipos: Exergames, Juegos deportivos y Juegos de aprendizaje, como se puede observar en el Cuadro 1 (GONZALEZ GONZÁLEZ; NAVARRO ADELANTADO, 2015).

Cuadro 1 - Categorías de videojuegos activos

\begin{tabular}{|l|l|}
\hline Categorías & Ejemplos \\
\hline Exergames & $\begin{array}{l}\text { Dance Dance Revolution, Wii Fit, GZ Endurance Cycle, } \\
\text { Sony Eye Toy }\end{array}$ \\
\hline Juegos deportivos & BrainBreaks, Sportwall XerTrainer, Makoto, LightSpace Wall \\
\hline Juegos de aprendizaje & FootPOWR, GZ Kids-Sport, NeuroActive BrainBike \\
\hline
\end{tabular}

Fuente: Elaboración propia.

Con determinados videojuegos y dispositivos, algunos de ellos ampliamente comercializados, jugar puede ser un ejercicio saludable y hacer ejercicio puede ser divertido. En este caso, algunos dispositivos se orientan a los ejercicios repetitivos de rehabilitación y otros simplemente a mejorar la forma física, como el Wii Fit. Algunos dispositivos comerciales, tales como la Wii o la Kinect, están siendo utilizados por la medicina para utilizarlos en la terapia ocupacional o en rehabilitación (CLARK et al, 2010). Por otra parte, encontramos diferentes tipos de software orientados a la salud, tales como los videojuegos para la prevención y promoción de la salud, otros para la mejora de la salud y por último, videojuegos destinados a la formación del personal sanitario (SERRANO, 2010).

La actividad física no solo tiene efectos fisiológicos y sobre la salud física, sino que también repercute positivamente sobre el bienestar psicológico del niño obeso, aumentando la autoestima, la satisfacción vital, y la adaptación social (formar parte de clubes o equipos favorece que se establezcan lazos de amistad). La investigación ha mostrado que los niños obesos tienen generalmente baja autoeficacia deportiva percibida e insatisfacción corporal, y que por esta razón suelen llevar una vida más sedentaria (SOUTHHALL; OKELY; STEELE, 2004). La primera dificultad que se encuentran los pediatras en la aplicación de la prescripción de hacer deporte en los niños y niñas con obesidad que están en tratamiento, es la falta de motivación. Es necesario, por tanto, establecer programas de actividad física adaptados a las condiciones de cada niño y niña, adaptando las metas, y el nivel de esfuerzo subjetivo, para evitar de esta manera que se sientan desmotivados, y abandonen la práctica (GUIXERES et al, 2011). Sin embargo, existen pocos estudios en la literatura que evalúen la eficacia real de la utilización de estos juegos como medio de favorecer la actividad física entre niños y adolescentes, y muy pocos que se centren en su utilización como parte del tratamiento en niños obesos probablemente porque se trata de un fenómeno relativamente nuevo. Aun así, los programas de intervención centrados en aumentar la actividad física son una forma efectiva de invertir el dinero público para prevenir la obesidad (MOYA MARTÍNEZ, 2011). 
En relación al mundo de los videojuegos y su uso como una aplicación para promover hábitos de vida saludable, destacan por su impacto e innovación algunos sistemas o plataformas que comentaremos seguidamente.

Let's Move! (¡A moverse!)2 es un programa integral creado por Michael Obama en 2010 para prevenir y tratar la obesidad infantil, en él se incluyó un concurso para premiar la mejor aplicación destinada a la salud infantil. El ganador fue el juego Smash your Food (Aplasta tu comida), donde literalmente el niño o niña simula aplastar la comida con un compresor para ver el contenido de sal, azúcar y grasa de diferentes alimentos.

Una aplicación similar de Medtronic, líder mundial en tecnología médica, es "Contando Carbohidratos con Lenny",3 un León que enseña a los niños con diabetes a aprender el contenido de carbohidratos de los alimentos.

Leap Frog es una de las compañías que fabrica juguetes digitales más conocidas a nivel mundial, esta empresa estadounidense fabrica LeapBand, ${ }^{4}$ una band donde los niños pueden interactuar con una mascota virtual, esta les obliga a mantenerse en movimientos mediante una serie de actividades y retos, y así fomentar un estilo de vida más saludable.

La empresa Hopelab, sin fines de lucro y orientada a potenciar la tecnología para mejorar la salud de los niños, creó Zamzee, que actualmente ha pasado a formar parte de WellTok.5 Zamzee es un programa para fomentar la actividad física entre los adolescentes, para ello premia la cantidad de ejercicio realizado con "Pointz" o Puntos (correr, saltar, pasear). Esta actividad física se registra con un dispositivo (acelerómetro) que deben llevar siempre encima, y que al conectarse al ordenador calcula la cantidad de puntos que has generado y que luego se pueden canjear por productos de las empresas que participan, desde personalizaciones de Zamzee hasta dispositivos iPod o donaciones. Los resultados aplauden la eficacia del producto

\footnotetext{
2 Página web del programa Let's Move: $<\mathrm{http} / /$ www.letsmove.gov/ en-espanol>.

3 Página de descarga en iTunes del programa Contando Carbohidratos con Lenny: $<$ https://itunes.apple.com/us/app/contando-carbohidratos-con/id464296487?mt=8>.

4 Página del producto Leapfrog: <http://www.leapfrog.com/en-us/ products/leapband>.

5 Página del producto Zamee: $<$ https://www.zamzee.com/>.
}

que en 6 meses ha visto como la actividad de los niños ha aumentado en un $59 \%$ respecto a sus otros compañeros.

Otro sistema muy parecido al anterior es el creado por la compañía GeoPalz, 6 iBitz está orientado a fomentar la actividad física a niños, y mide la cantidad de pasos que dan los niños mediante un podómetro. Con él los niños pueden ver los pasos que han dado e introducirlos en la web. Los pasos se convierten en puntos canjeables por regalos en diferentes portales que los padres han seleccionado previamente y a los que han puesto un precio en puntos. Además, el número de puntos recogidos durante el día van a comprar unos minutos de juego, cuando expiren, el juego se cierra hasta que el niño se mueva un poco más. GeoPalz se ha asociado con desarrolladores de juego de manera que los puntos del niño puedan ser utilizados para conseguir secretos y logros en unos juegos determinados.

En Brasil, también se han desarrollado programas de intervención y estudios con exergames, como por ejemplo, el proyecto Criança em Movimento.7 En este sentido, existe un exergame denominado Peggo, específicamente diseñado por la Universidad Federal de Pernambuco para combatir la obesidad infantil, en el que se utiliza una cámara web como una herramienta de interacción entre el usuario y el sistema (BARROS et al, 2013). En Canarias, se ha desarrollado una plataforma de realización de juegos activos basada en el sensor Kinect de Microsoft (c) denominado TANGO:H (Tangible Goals: Health) (GONZALEZ et al, 2013). La potencia de TANGO:H reside en su capacidad de generación de ejercicios, es decir, no es una plataforma estática en la que los ejercicios o juegos están plenamente definidos e integrados, sino que permite la implementación de estos mediante un configurador que hace sencilla esta tarea. El programa es capaz de interpretar y ejecutar los ejercicios previamente creados por un fisioterapeuta o especialista en educación en el editor Tango: $\mathrm{H}$ Designer (Tangible Goals: Health Designer). El usuario realiza los ejercicios previamente creados en forma de un videojuego, interactuando con el sistema a través de movimientos corporales y ges-

6 Página web del producto Geopalz: $<$ http://geopalz.com/>.

7 Proyecto "Criança em Movimento": <http://projetocriancaemmovimento.blogspot.ca/2012/06/videogame-pode-ajudar-combater. html>. 
tos. La combinación entre el editor y los módulos de juego permite la creación de una gran variedad de ejercicios, personalizados y adaptados a las características de los usuarios. La aplicación posee una interfaz sencilla que guía al usuario en la selección y ejecución de los ejercicios disponibles.

Lo Cuadro 2 muestra un ejemplo de diseño de un ejercicio para una sesión con TANGO:H Designer.

Cuadro 2 - Ejemplo de ejercicio de una sesión usando TANGO

\section{Sesión "Pirámide de la actividad física"}

\section{Ejercicio 1}

El jugador debe emparejar las diferentes actividades que le aparecen a la izquierda con la posición de la pirámide de la actividad física que corresponda.
Título: Une cada actividad en el lugar de la pirámide que corresponda.

Tipo de ejercicio: Cognitivo de emparejamiento

Modo de juego: Colaborativo

Número de pasos: 2

Número total de objetivos alcanzables: 16

Número total de distractores: 0

Duración máxima para máxima puntuación: 56 segundos

Máximo de puntos: 320

Mínimo de puntos: 16

Normas especiales: Se les pide a la pareja de jugadores que se coja del brazo y que sólo pueden tocar los objetivos con las manos y piernas libres.

Paso 1

Número de fases: 4

Número de objetivos alcanzables: 8

Número de distractores
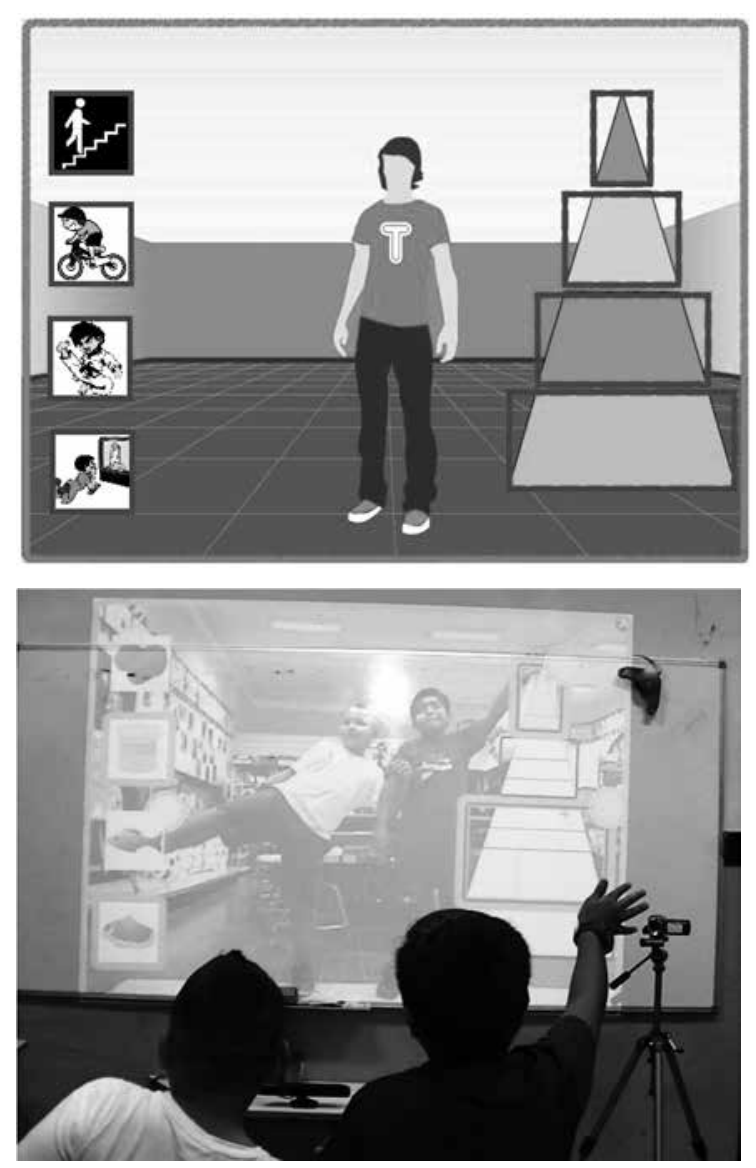

Fuente: Elaboración propia. 
Haunque algunos estudios han mostrado resultados positivos acerca de los costes energéticos de jugar a este tipo de juegos, este coste energético se eleva con la realización de esas mismas actividades virtuales en la realidad, indicando que este tipo de actividad no puede ser un sustituto de las actividades y deportes reales. Solo un pequeño número de videojuegos activos o "exergaming" consiguen que el niño realice una actividad física de intensidad moderada. A esto se le une, que hasta la fecha son muy pocos los estudios que tratan de estudiar los diferentes efectos de los videojuegos activos en niños y niñas con obesidad (FALCADE et al, 2013) y aún existen muy pocas investigaciones relacionadas con la aplicación de los videojuegos activos en la rehabilitación. Por lo tanto, se necesitan más estudios de mayor evidencia científica para evaluar la efectividad y sostenibilidad de este tipo de videojuegos activos así como su posible interés como herramienta clínica.

En este sentido, destacaremos el estudio "Valoración de la intervención formativa con videojuego activo y juego motor en niños" cuyo objetivo general era educar a los menores en hábitos de vida saludable a través de juegos motores y videojuegos activos. Esta intervención se llevó a cabo en el Colegio de San Fernando Duggi de Santa Cruz de Tenerife en un grupo seleccionado de 20 escolares de entre 8 y 12 años de edad, unos participaron en el grupo control y otros en el experimental. La intervención se realizó en 16 sesiones durante todo de octubre a diciembre de 2012 donde se trabajaban temas sobre hábitos de vida saludable realizando, en el colegio y en sus hogares, diferentes juegos motores y participando en juegos grupales con los videojuegos TANGO-H (Kinect) (GONZALEZ et al, 2013), isla SAVEH (Ordenador) (GONZÁLEZ-GONZÁLEZ; BLANCO-IZQUIERDO, 2012) y el Wii Fit Plus (Consola Wii). Además, los padres y madres también recibieron una sesión formativa sobre hábitos de vida saludables y videojuegos. Los resultados de dicha investigación demuestran la eficacia del programa formativo de estas características para promover el cambio de conductas relacionadas con los hábitos de vida saludables (GONZÁLEZ et al, 2016). El proyecto PROVITAO, que se describe a continuación, tiene sus orígenes en esta investigación.

\section{Provitao: programa de intervención educativa para el tratamiento de la obesidad infantil}

PROVITAO es un programa de intervención educativa utilizando videojuegos activos y juegos motores para el apoyo al tratamiento ambulatorio (en domicilio) de la obesidad infantil, que se propone mejorar la adhesión y cumplimiento terapéutico y la adquisición de hábitos saludables en menores y su entorno familiar. Es un proyecto desarrollado entre el Grupo de Investigación de Interacción, Tecnologías y Educación (ITED) de la Universidad de La Laguna y cuenta con la financiación de la Fundación CajaCanarias, en el marco del Programa de Ayudas para proyectos de Investigación Sanitaria, y con la colaboración del Servicio Canario de Salud, del Hospital Universitario de Canarias (HUC) y el Instituto de Tecnologías y Energías Renovables (ITER).

El objetivo principal del proyecto es validar la efectividad del modelo de intervención basado en juegos motores y videojuegos activos en el cambio de actitudes y hábitos saludables en niños con obesidad en un primera fase y con obesidad y diabetes tipo II en una segunda fase en comparación con el modelo actual de tratamiento que se lleva a cabo en las consultas médicas. Además de servir como herramienta para la prevención y promoción de la salud en estilos de vida saludables. Se cuenta para ello con un programa de intervención educativa para la educación en hábitos saludables. Dicho modelo es el que se plantea en este trabajo, junto a un programa de ejercicios, juegos motores y videojuegos comerciales y propios del grupo, tales como TANGO:H, para los pacientes y para los padres.

\subsection{Diseño del programa de intervención}

La intervención educativa que se presenta a continuación pretende ser una herramienta eficaz que favorece la adquisición de hábitos saludables en los niños y niñas y sus familias, y que repercuta en la mejora de la calidad de vida, actual y futura, de los menores que padecen obesidad infantil. De esta forma, los objetivos que persigue son los siguientes: 
- Promover la adquisición y permanencia de hábitos de vida saludables en niños y niñas con sobrepeso/obesidad a través de la educación para la salud.

- Evaluar la influencia del programa de intervención educativa en menores con sobrepeso/obesidad.

- Promover una conciencia social sobre la importancia del desarrollo de la obesidad infantil (los problemas, las complicaciones a largo plazo, los costes sanitarios y sociales) y la prevención en este ámbito.

La metodología del estudio es cuasi-experimental, de corte longitudinal y prospectivo de 3 años de duración (desde marzo de 2014 hasta julio de 2017). Consta de dos fases, y en cada fase se dispone de un grupo control y un grupo experimental. El programa se realiza en el Hospital Universitario de Canarias (HUC), ubicado en la provincia de Santa Cruz de Tenerife, Comunidad Autónoma de Canarias, España, un centro asistencial dependiente de la Consejería de Sanidad del Gobierno de Canarias, orientado a la asistencia sanitaria de la zona norte de Tenerife, y que es hospital de referencia para la isla de La Palma. Para llevar a cabo el trabajo de campo y recogida de datos, se ha solicitado el permiso al Comité ético de investigación del HUC.

La realización de este trabajo ha seguido las directrices y principios éticos para las investigaciones médicas en seres humanos, según se recoge en la Declaración de Helsinki adoptada en la Asamblea de la Asociación Médica Mundial (AMM) en 1964, y en la última actualización de 2004. Se asegura por lo tanto, el conocimiento y aprobación por parte del Comité ético de Hospital Universitario de Canarias en calidad de entidad responsable, así como los propios padres o tutores responsables de los alumnos.

Se garantiza, además el anonimato de los participantes así como la confidencialidad de la información, siendo estos datos recabados utilizados únicamente con fines científico.
Se han realizado consentimientos informados diferenciados para los padres o tutores legales y para la participación de los niños. La participación es voluntaria, sin embargo, la aceptación de la participación implica el compromiso de colaboración de los padres y los niños. En la hoja de información se especifica el tipo de intervención y número de visitas que van a realizarse, así como el lugar donde tendrán que acudir y el número de horas que se dedicarán a la intervención y qué parámetros se van a valorar. También se explica que el período del estudio coincide con el curso escolar, la existencia de los diferentes grupos, la necesidad de ser grabados en vídeo para el análisis de datos y el cumplimiento de la ley de protección de datos.

Estas hojas informativas son claras evitando el uso de acrónimos para facilitar que los pacientes entiendan la información que se le está transmitiendo.

Aquellos niños y niñas que abandonen el estudio, no podrán ser reemplazados, ya que dadas las características del estudio, no se podría valorar el efecto del conjunto de la intervención.

La población diana son los niños y niñas diagnosticados de obesidad/diabetes tipo II vistos en las consultas externas de Pediatría del HUC. El número de niños obesidad que acuden al área de consultas de Pediatría es de 50 niños/ día. La muestra se compone de 40 niños y niñas de entre [8-12] años de edad cuyo IMC sea superior al P97 (indicador de obesidad infantil).

Los niños y niñas acuden a la consulta de pediatría del HUC y en la consulta se les invita y se les explica brevemente el objetivo del estudio. El médico realiza la primera recogida de variables, para confirmar la existencia de obesidad en el menor. En la consulta, se hace entrega de un documento informativo sobre el proyecto, con un pequeño cuestionario para saber si cumplen los criterios de inclusión o de exclusión del proyecto (Tabla 3). Posteriormente, en la reunión informativa se hace entrega de los consentimientos informados, para obtener acceso a los datos clínicos. 
Cuadro 3 - Criterios de inclusión y exclusión del estudio

\section{Criterios}

\begin{tabular}{|l|l|}
\hline \multirow{5}{*}{ Inclusión } & $\begin{array}{l}\text {-Niños/as entre } 8 \text { y } 12 \text { años con obesidad utilizando como criterio un } \\
\text { IMC superior al Pc } 97 \text { para la primera fase del proyecto. } \\
\text {-Niños/as entre } 8 \text { y } 12 \text { años con obesidad utilizando como criterio } \\
\text { un IMC superior al Pc } 97 \text { y Diabetes tipo II para la segunda fase del } \\
\text { proyecto. } \\
\text {-Pacientes que acudan al servicio de Pediatría del HUC. }\end{array}$ \\
\hline Exclusión & $\begin{array}{l}\text {-Niños y niñas que no cuenten con tecnologías de redes básicas en } \\
\text { casa (ordenador e internet) y televisor. El proyecto se encargará de } \\
\text { proporcionar el resto de herramientas tecnológicas requeridas para la } \\
\text { intervención en el domicilio (sensor Kinect, consola Wii y Wii balance } \\
\text { board) y en las sesiones grupales. } \\
\text {-Niños y niñas cuyos padres no deseen participar en el proyecto. } \\
\text {-Niños y niñas con deterioro cognitivo que impide participar en el } \\
\text { proyecto. } \\
\text {-Participación durante los últimos } 12 \text { meses en un ensayo clínico. }\end{array}$ \\
\hline
\end{tabular}

Fuente: Elaboración propia.

Las variables e instrumentos de medida que se utilizan en el proyecto se dividen en función del área de análisis, las cuales se describen en lo Cuadro 4.

Cuadro 4 - Medidas, instrumentos y momentos de la intervención por área disciplinar implicada

\begin{tabular}{|c|c|c|c|}
\hline Áreas & Medidas & Instrumento utilizado & $\begin{array}{l}\text { Momento } \\
\text { de medida }\end{array}$ \\
\hline Médica & $\begin{array}{l}\text {-Peso } \\
\text {-Estatura } \\
\text {-Pliegues cutáneos } \\
\text {-Diámetros óseos } \\
\text {-Perímetros musculares y corporales } \\
\text {-Composición corporal e índice de } \\
\text { masa corporal } \\
\text {-Medidas fisiológicas } \\
\text {-Esfuerzo percibido }\end{array}$ & $\begin{array}{l}\text { Pesa } \\
\text { Altímetro } \\
\text { Plicómetro } \\
\text { Compás } \\
\text { Cinta métrica } \\
\text { Fórmula (según método } \\
\text { tetracompartimental y } \\
\text { somatotipo) } \\
\text { Pulsímetro y acelerómetro } \\
\text { Escala del esfuerzo } \\
\text { percibido }\end{array}$ & $\begin{array}{l}\text { Sesiones } \\
\text { (pre, } \\
\text { durante y } \\
\text { post) }\end{array}$ \\
\hline $\begin{array}{l}\text { Lúdico - } \\
\text { emocional }\end{array}$ & $\begin{array}{l}\text {-Estado emocional en el juego motor } \\
\text { y con el videojuego }\end{array}$ & $\begin{array}{l}\text { Emodiana (GONZÁLEZ; } \\
\text { NAVARRO- } \\
\text { ADELANTADO; CAIROS, } \\
\text { 2013) }\end{array}$ & $\begin{array}{l}\text { Sesiones } \\
\text { (pre y post) }\end{array}$ \\
\hline
\end{tabular}




\begin{tabular}{|c|c|c|c|}
\hline Áreas & Medidas & Instrumento utilizado & $\begin{array}{l}\text { Momento } \\
\text { de medida }\end{array}$ \\
\hline Interactividad & $\begin{array}{l}\text {-Perfil del jugador } \\
\text {-Uso de videojuego TANGO-H } \\
\text {-Experiencia usuario TANGO-H } \\
\text {-Actitud hacia los videojuegos }\end{array}$ & $\begin{array}{l}\text {-Entrevista de perfil de } \\
\text { jugador } \\
\text {-Grabación de videos } \\
\text { - Registros de TANGO-H } \\
\text {-Cuestionario de experiencia } \\
\text { usuario } \\
\text {-Adaptación del } \\
\text { Cuestionario sobre uso } \\
\text { y actitudes hacia los } \\
\text { videojuegos (ALFAGEME; } \\
\text { SÁNCHEZ, 2003) }\end{array}$ & $\begin{array}{l}\text { - Pre } \\
\text { - Sesiones } \\
\text { - Post } \\
\text { - Pre y post }\end{array}$ \\
\hline $\begin{array}{l}\text { Psicología y } \\
\text { pedagogía }\end{array}$ & $\begin{array}{l}\text {-Relaciones interpersonales, } \\
\text { relaciones con los padres, } \\
\text { autoestima, confianza en sí mismo } \\
\text { (NIÑOS y NIÑAS) } \\
\text {-Conocimientos sobre hábitos de } \\
\text { vida saludables, hábitos y actitudes } \\
\text { hacia la Actividad Física y la } \\
\text { alimentación (NIÑOS y NIÑAS) } \\
\text { - Conocimientos sobre hábitos de } \\
\text { vida saludables, hábitos y actitudes } \\
\text { hacia la Actividad Física y la } \\
\text { alimentación (PADRES/MADRES) } \\
\text {-Índice de calidad de la dieta } \\
\text { mediterránea } \\
\text {-Satisfacción con la formación de los } \\
\text { padres }\end{array}$ & $\begin{array}{l}\text {-BASC (Sistema de } \\
\text { evaluación de la conducta } \\
\text { de niños y adolescentes) } \\
\text { - Cuestionario propio } \\
\text { - Cuestionario propio } \\
\text { - KidMed } \\
\text { - Cuestionario propio }\end{array}$ & - Pre y post \\
\hline
\end{tabular}

Fuente: Elaboración propia.

La intervención educativa se desarrolla en tres fases diferenciadas, las cuales se describen a continuación:

$1^{\circ}$ Fase: Evaluación pre-intervención. En esta etapa inicial se procede a la solicitud de permisos y a la preparación del trabajo de campo. Teniendo en cuenta el tipo de estudio, la población, muestra, variables e instrumentos, y los métodos de recogida y de análisis de la información relevante para la realización del estudio. Se realiza además un estudio descriptivo transversal de la muestra, siguiendo los objetivos siguientes:

- Valorar el entorno, los factores de riesgo, los factores protectores, los hábitos no saludables, y el nivel de conocimiento que tienen los niños, y sus familias;

- Conocer la prevalencia de la obesidad y del sobrepeso de la muestra.

$2^{\circ}$ Fase: Desarrollo de la intervención. En esta fase se realiza un estudio analítico observacional longitudinal. Esta fase se organiza en los trimestres del curso escolar y se repite 2 años. Durante esta fase del estudio se lleva a cabo una intervención educativa y promocional sobre los hábitos y estilos de vida saludables a los menores participantes y sus familias. 
Los objetivos a conseguir son:

- Proporcionar información sobre la importancia y necesidad de una alimentación adecuada durante las primeras etapas de vida, para prevenir la aparición de obesidad.

- Conocer la importancia tanto a nivel social como sanitario de padecer obesidad.

- Fomentar hábitos y estilos de vida saludables, promoviendo un cambio de conducta permanente.

- Desarrollar la autoestima individual, fomentar la comunicación y relación entre compañeros y con sus familias, crear un entorno familiar que refuerce los buenos hábitos para mejorar la calidad de vida de los pacientes con obesidad.

Se utiliza el juego social y activo como estrategia para la motivación y fomento de actitudes, capacidades y conocimiento de sí mismo, así como se ayudará a que los menores desarrollen actividades saludables que partan de sus propios intereses a través de un programa vocacional.

La intervención durante el primer trimestre del curso escolar se organiza de la siguiente manera:

1. Realización de sesiones grupales para niños y niñas. Durante las 12 semanas (3 meses) de la intervención grupal con juego motor, los participantes deberán acudir a sesiones grupales de 2 horas de duración una vez por semana, en las que hacen una actividad formativa de hábitos saludables y educación sanitaria sobre obesidad/diabetes (60 minutos), practican juegos motores tradicionales y realizan en parejas actividades de un videojuego activo con contenidos en hábitos saludables (60 minutos);

2. Realización de sesiones en domicilio para niños y niñas. Además deben realizar otras actividades semanales programadas para el domicilio, donde practicarán 30 minutos de videojuego comercial con contenido de actividad física (Wii Fit Plus);

3. Realización de actividad formativa para padres y madres.

Para los niños y niñas participantes se desarrolla un programa educativo, donde se establecen distintos talleres o sesiones formativas de 60 minutos de duración, que se imparten una vez a la semana.
Los temas que se imparten son:

- Los alimentos y los nutrientes. Contenido energético de los diferentes grupos de alimentos. Pirámide de alimentos;

- Patrones alimentarios del niño y del adolescente. Dificultades más comunes en relación con la alimentación a estas edades;

- Trastornos alimentarios en niños y del adolescente. Factores de riesgo;

- Valoración del estado nutricional, y percepción de la imagen corporal;

- Eliminar mitos sobre los alimentos y las dietas;

- Características de una dieta sana y equilibrada. Dieta mediterránea;

- Consejos para elegir la forma de preparar y cocinar;

- MasterChef Junior;

- El carrito de la compra;

- Ingesta emocional;

- Actividad física como hábito de vida saludable. Beneficios para la vida;

- Videojuegos. Mitos y creencias.

Para las distintas actividades que realizaremos con los niños y niñas se utilizan métodos expositivos y participativos mediante la dinámica de grupos, el uso de debates y juego de rol.

Para los padres y madres, se estable una sola sesión educativa de 90 minutos de duración, donde se tratan tres temas: los hábitos de vida saludables, la obesidad como una enfermedad, y las falsas creencias sobre los videojuegos.

La intervención durante el segundo trimestre curso escolar se organiza de la siguiente manera:

1. Realización de sesiones en domicilio para niños y niñas. Durante las siguientes 12 semanas (3 meses), la intervención será individual y domiciliaria, apoyadas en TIC. Como herramientas TIC se utilizarán la videoconferencia y un videojuego comercial (Wii Fit Plus);

2. Creación de un proyecto vocacional. Se trabaja en la creación por los propios menores de un proyecto vocacional en relación al descubrimiento de actividades saludables que puedan gustarles y a su entorno. Esta actividad es tutorizada por un miembro del equipo de investigación a través de una se- 
sión de videoconferencia semanal. Asimismo, se sigue con el programa de actividades con la Wii Fit Plus en el domicilio;

3. Contacto con los ayuntamientos, asociaciones, etc. de la zona de los menores para conocer los recursos y actividades saludables disponibles;

4. Realización de una actividad de orientación para padres y madres. Este trimestre incluye una actividad de orientación para padres y madres sobre la orientación vocacional de sus hijos/hijas.

Por último, la intervención durante tercer trimestre curso escolar se realiza de la siguiente forma:

1. Realización del proyecto vocacional. Durante las últimas 12 semanas (3 meses), la intervención será individual y domiciliaria, apoyadas en TIC. Como herramientas TIC se utiliza la videoconferencia. El objetivo de esta intervención trimestral es apoyar a los menores en el desarrollo de su propio proyecto vocacional en relación a las actividades saludables. Esta actividad es tutorizada por un miembro del equipo de investigación a través de una sesión de videoconferencia semanal;

2. Realización de una actividad de asesoramiento para padres y madres. Este trimestre incluye una actividad de asesoramiento para padres y madres sobre el apoyo al desarrollo del proyecto vocacional de sus hijos/hijas.
Dicha intervención se lleva a cabo en un espacio de un centro educativo, dotado de las infraestructuras necesarias, $y$ en horario de tarde para no interferir en la vida escolar de los participantes.

\section{$3^{\circ}$ Fase: Evaluación}

- En esta fase se desea evaluar la influencia del programa de intervención educativa en menores con obesidad y validar la eficacia de las herramientas tecnológicas desarrolladas como soporte al tratamiento. Así que, cada 6 meses según consulta médica, y durante el siguiente año, se realizan evaluaciones antropométricas de los niños y niñas de ambos grupos (tanto experimental como control).

\subsection{Valoración parcial del programa de intervención}

Actualmente se están analizando los datos de los cursos en los que se ha realizado la intervención para medir la efectividad del programa en todas las áreas (médica, psicológica, interactiva, etc.). Sin embargo, podemos ya medir el grado de satisfacción respecto a los juegos de los participantes del programa a través del cuestionario de satisfacción que hemos utilizado con el grupo experimental del curso 2014-2015, quienes ya han finalizado la intervención. La tabla X muestra algunas respuestas de los12 niños y niñas del grupo experimental frente a las preguntas realizadas sobre el programa de intervención, centradas en los juegos motores, videojuegos activos y el uso de la Wii en el domicilio.

Cuadro 5 - Algunas preguntas del cuestionario de satisfacción del programa de intervención para los niños y niñas del grupo experimental.

\begin{tabular}{|l|l|l|l|l|l|}
\hline \multirow{2}{*}{ Preguntas } & \multicolumn{3}{l|}{ Resultados } \\
\hline \multirow{2}{*}{ 1. ¿Ibas con ganas a las sesiones semanales? } & $\begin{array}{l}1 \\
\text { (nada) }\end{array}$ & 2 & 3 & 4 & $\begin{array}{l}5 \\
\text { (mucho) }\end{array}$ \\
\cline { 2 - 7 } & $0 \%$ & $0 \%$ & $16,7 \%$ & $25 \%$ & $58,3 \%$ \\
\hline \multirow{2}{*}{ 2. ¿Te divertías en las sesiones semanales? } & $\begin{array}{l}1 \\
\text { (nada) }\end{array}$ & 2 & 3 & 4 & $\begin{array}{l}5 \\
\text { (mucho) }\end{array}$ \\
\cline { 2 - 7 } & $0 \%$ & $0 \%$ & $0 \%$ & $33,3 \%$ & $66,7 \%$ \\
\hline
\end{tabular}




\begin{tabular}{|c|c|c|c|c|c|}
\hline 3. ¿Qué te gustaba de las sesiones semanales? & \multicolumn{5}{|c|}{$\begin{array}{l}\text { Los juegos y estar con los compañeros. } \\
\text { Porque me divertía juegos. } \\
\text { Mejorar en mis sesiones y divertirme mucho. } \\
\text { Que aprendía cosas nuevas y me divertía con } \\
\text { todas las. } \\
\text { Jugar } \\
\text { Que jugaba y sudaba y me divertía mucho y } \\
\text { además, aprendía. } \\
\text { Lo juegos } \\
\text { Todo lo que hacíamos. } \\
\text { Los juegos eran divertidos, } \\
\text { Que me divertía y tenía más amigos para jugar. } \\
\text { Tango: H. } \\
\text { Los juegos que hacíamos. }\end{array}$} \\
\hline 4. ¿Qué te animaba más a ir? & \multicolumn{5}{|c|}{$\begin{array}{l}\text { Lo que aprendía }(25 \%) \\
\text { Jugar }(33.3 \%) \\
\text { Hacer actividad física }(16.7 \%) \\
\text { Ganar puntos para la clasificación }(0 \%) \\
\text { Estar con los compañeros }(8.3 \%) \\
\text { Estar con los profesionales }(8.3 \%) \\
\text { Mejorar mi estilo de vida }(8.3 \%) \\
\text { Otro }(0 \%)\end{array}$} \\
\hline 5. ¿Qué otras cosas que también te animaban a ir? & \multicolumn{5}{|c|}{$\begin{array}{l}\text { Lo que aprendía }(50 \%) \\
\text { Jugar }(58.3 \%) \\
\text { Hacer actividad física }(25 \%) \\
\text { Ganar puntos para la clasificación }(41.7 \%) \\
\text { Estar con los compañeros }(50 \%) \\
\text { Estar con los profesionales }(8.3 \%) \\
\text { Mejorar mi estilo de vida }(25 \%) \\
\text { Otro }(0 \%)\end{array}$} \\
\hline $\begin{array}{l}\text { 6. ¿Qué es lo que menos te gustaba de las sesiones } \\
\text { semanales? }\end{array}$ & \multicolumn{5}{|c|}{$\begin{array}{l}\text { Nada. } \\
\text { La emodiana } \\
\text { Nada } \\
\text { Me gustaba todo. } \\
\text { Nada, me gustaba todo. } \\
\text { Algunas cosas: la formación } \\
\text { Estar sentada en el suelo. }\end{array}$} \\
\hline \multirow{2}{*}{ 7. ¿Cuánto te gustaba la formación? } & $\begin{array}{l}1 \\
\text { (nada) }\end{array}$ & 2 & 3 & 4 & $\begin{array}{l}5 \\
\text { (mucho) }\end{array}$ \\
\hline & $0 \%$ & $0 \%$ & $25 \%$ & $41,7 \%$ & $33,3 \%$ \\
\hline \multirow{2}{*}{ 8. ¿Cuánto te gustaban los juegos de la formación? } & $\begin{array}{l}1 \\
\text { (nada) }\end{array}$ & 2 & 3 & 4 & $\begin{array}{l}5 \\
\text { (mucho) }\end{array}$ \\
\hline & $0 \%$ & $0 \%$ & $0 \%$ & $50 \%$ & $50,3 \%$ \\
\hline \multirow{2}{*}{ 9. ¿Cuánto te gustaban los juegos en la cancha? } & $\begin{array}{l}1 \\
\text { (nada) }\end{array}$ & 2 & 3 & 4 & $\begin{array}{l}5 \\
\text { (mucho) }\end{array}$ \\
\hline & $0 \%$ & $0 \%$ & $8,3 \%$ & $25 \%$ & $66,7 \%$ \\
\hline
\end{tabular}




\begin{tabular}{|c|c|c|c|c|c|}
\hline \multirow{2}{*}{ 10. ¿Cuánto te gustaba jugar con TANGO:H? } & $\begin{array}{l}1 \\
\text { (nada) }\end{array}$ & 2 & 3 & 4 & $\begin{array}{l}5 \\
\text { (mucho) }\end{array}$ \\
\hline & $0 \%$ & $0 \%$ & $16,7 \%$ & $41,7 \%$ & $41,7 \%$ \\
\hline \multirow{2}{*}{ 11. ¿Cuánto te gustaba pensar en cómo te sentías? } & $\begin{array}{l}1 \\
\text { (nada) }\end{array}$ & 2 & 3 & 4 & $\begin{array}{l}5 \\
\text { (mucho) }\end{array}$ \\
\hline & $8,3 \%$ & $0 \%$ & $58,3 \%$ & $0 \%$ & $33,3 \%$ \\
\hline \multirow{2}{*}{$\begin{array}{l}\text { 12. ¿Resultaba incómodo llevar puesto el } \\
\text { pulsómetro y el podómetro? }\end{array}$} & $\begin{array}{l}1 \\
\text { (nada) }\end{array}$ & 2 & 3 & 4 & $\begin{array}{l}5 \\
\text { (mucho) }\end{array}$ \\
\hline & $33,3 \%$ & $33,3 \%$ & $8,3 \%$ & $16,7 \%$ & $8,3 \%$ \\
\hline \multirow{2}{*}{$\begin{array}{l}\text { 13. ¿Te sentías bien haciendo los juegos en la } \\
\text { cancha? }\end{array}$} & $\begin{array}{l}1 \\
\text { (nada) }\end{array}$ & 2 & 3 & 4 & $\begin{array}{l}5 \\
\text { (mucho) }\end{array}$ \\
\hline & $0 \%$ & $0 \%$ & $16,7 \%$ & $16,7 \%$ & $66,7 \%$ \\
\hline \multirow{2}{*}{ 14. ¿Te sentías bien jugando con Tango:H? } & $\begin{array}{l}1 \\
\text { (nada) }\end{array}$ & 2 & 3 & 4 & $\begin{array}{l}5 \\
\text { (mucho) }\end{array}$ \\
\hline & $0 \%$ & $0 \%$ & $8,3 \%$ & $33,3 \%$ & $58,3 \%$ \\
\hline \multirow{2}{*}{$\begin{array}{l}\text { 15. ¿Te gustaba hacer las sesiones de actividad física } \\
\text { con la Wii en casa? }\end{array}$} & $\begin{array}{l}1 \\
\text { (nada) }\end{array}$ & 2 & 3 & 4 & $\begin{array}{l}5 \\
\text { (mucho) }\end{array}$ \\
\hline & $0 \%$ & $0 \%$ & $33,3 \%$ & $8,3 \%$ & $58,3 \%$ \\
\hline 16. ¿Qué te animaba más para hacer la Wii en casa? & \multicolumn{5}{|c|}{$\begin{array}{l}\text { Era divertido }(58.3 \%) \\
\text { Hacer actividad física }(25 \%) \\
\text { Ganar puntos para la clasificación }(0 \%) \\
\text { Que era obligatorio } \quad(8.3 \%) \\
\text { Otro } \quad(8.3 \%)\end{array}$} \\
\hline \multirow{2}{*}{$\begin{array}{l}\text { 17. ¿Has aprendido cosas nuevas durante las } \\
\text { sesiones de formación? }\end{array}$} & $\begin{array}{l}1 \\
\text { (nada) }\end{array}$ & 2 & 3 & 4 & $\begin{array}{l}5 \\
\text { (mucho) }\end{array}$ \\
\hline & $0 \%$ & $0 \%$ & $16,7 \%$ & $41,7 \%$ & $41,7 \%$ \\
\hline \multirow{2}{*}{ 18. ¿Crees que lo que has aprendido es útil? } & $\begin{array}{l}1 \\
\text { (nada) }\end{array}$ & 2 & 3 & 4 & $\begin{array}{l}5 \\
\text { (mucho) }\end{array}$ \\
\hline & $8,3 \%$ & $0 \%$ & $25 \%$ & $25 \%$ & $41,7 \%$ \\
\hline 19. ¿Recomendarías el programa a un amigo? & \multicolumn{5}{|c|}{$\begin{array}{l}\text { Sí } 91,7 \%(11) \\
\text { No } 8,3 \%(1)\end{array}$} \\
\hline
\end{tabular}




\begin{tabular}{|c|c|c|c|c|c|}
\hline \multirow{2}{*}{ 20. ¿Fue divertido usar la WII? } & $\begin{array}{l}1 \\
\text { (nada) }\end{array}$ & 2 & 3 & 4 & $\begin{array}{l}5 \\
\text { (mucho) }\end{array}$ \\
\hline & $0 \%$ & $0 \%$ & $16,7 \%$ & $8,3 \%$ & $75 \%$ \\
\hline 21. ¿Qué te animaba más a usar la WII? & \multicolumn{5}{|c|}{$\begin{array}{l}\text { Que era divertido }(58.3 \%) \\
\text { Hacer actividad física }(25 \%) \\
\text { Ganar puntos para la clasificación }(8.3 \%) \\
\text { Que era obligatorio }(8.3 \%) \\
\text { Otro } \quad(0 \%)\end{array}$} \\
\hline \multirow{2}{*}{ 22. ¿Te llegaste a aburrir de usar la WII? } & $\begin{array}{l}1 \\
\text { (nada) }\end{array}$ & 2 & 3 & 4 & $\begin{array}{l}5 \\
\text { (mucho) }\end{array}$ \\
\hline & $50 \%$ & $16,7 \%$ & $8,3 \%$ & $8,3 \%$ & $16,7 \%$ \\
\hline $\begin{array}{l}\text { 23. Si te aburriste ¿Qué fue lo que te aburrió o } \\
\text { desmotivó? }\end{array}$ & \multicolumn{5}{|c|}{$\begin{array}{l}\text { Jugar siempre el mismo juego (Fit plus) }(50 \%) \\
\text { Jugar solo }(37.5 \%) \\
\text { Que era obligatorio } \\
\text { Que no mejoraba mi puntuación }(0 \%) \\
\text { Que los ejercicios eran difíciles }(0 \%) \\
\text { Otro }(12.5 \%)\end{array}$} \\
\hline \multirow{2}{*}{$\begin{array}{l}\text { 24. ¿Crees que has aprendido algo sobre hábitos de } \\
\text { vida saludable? }\end{array}$} & $\begin{array}{l}1 \\
\text { (nada) }\end{array}$ & 2 & 3 & 4 & $\begin{array}{l}5 \\
\text { (mucho) }\end{array}$ \\
\hline & $0 \%$ & $0 \%$ & $8,3 \%$ & $25 \%$ & $66,7 \%$ \\
\hline \multirow{2}{*}{$\begin{array}{l}\text { 25. ¿Crees que la actividad física del programa ha } \\
\text { cambiado tu forma de comportarte con respecto a } \\
\text { tus hábitos de actividad física? }\end{array}$} & \multicolumn{3}{|l|}{ SI } & \multicolumn{2}{|l|}{ NO } \\
\hline & \multicolumn{3}{|l|}{$91,7 \%$} & \multicolumn{2}{|l|}{$8,3 \%$} \\
\hline \multirow{2}{*}{$\begin{array}{l}\text { 26. ¿Crees que la alimentación equilibrada ha } \\
\text { cambiado tu forma de comportarte con respecto a } \\
\text { tus hábitos de alimentación? }\end{array}$} & \multicolumn{3}{|l|}{ SI } & \multicolumn{2}{|l|}{ NO } \\
\hline & \multicolumn{3}{|l|}{$90,9 \%$} & \multicolumn{2}{|l|}{$9,1 \%$} \\
\hline
\end{tabular}

Fuente: Elaboración propia.

\section{Conclusiones}

En este artículo se ha presentado un análisis sobre la problemática de la obesidad infantil y su posible prevención y tratamiento utilizando videojuegos activos (exergames). Asimismo, se han analizado algunos programas y estudios relevantes que comparten el enfoque del proyecto PROVITAO, esto es, el utilizar los videojuegos activos como eje central de la intervención educativa.

Como aporte principal se ha presentado el diseño del programa de intervención educativa basada en juegos motores y videojuegos activos del proyecto PROVITAO. El mismo consta de diversas fases de intervención, y cada una de ellas tiene una serie de pasos, instrumentos, medidas y variables que han sido cuidadosamente seleccionadas y elaboradas por el propio equipo de investigación. Este proyecto tiene un enfoque interdisciplinar, por lo que el diseño y la toma de las medidas y variables utilizadas se realizan por cada área en particular, así como el análisis de los resultados.

La intervención ha tenido un desarrollo satisfactorio en cuanto al cumplimiento del programa de juegos motores y los videojuegos activos, tanto en las sesiones grupales como en las casas. Para 
valorar el grado de satisfacción del programa de intervención educativa desarrollado, se han presentado los resultados parciales del cuestionario de satisfacción de los niños y niñas del grupo experimental. La respuesta de los niños ha sido positiva a la propuesta y desarrollo del programa. Podemos observar que el $83,3 \%$ de los niños y niñas iban animados a las sesiones semanales y se divertían en las mismas (100\%). Sin duda, en las respuestas de los niños y niñas se observa que lo que más valoraban en las sesiones grupales es el juego en todas sus formas, en los juegos de formación (100\%), los juegos de la cancha $(82,7 \%)$ y TANGO:H $(83,4 \%)$. Sin embargo, no se sienten muy cómodos a la hora de valorar y expresar sus emociones. Con respecto a las sesiones en domicilio y el uso de la WII, aunque consideran que se han divertido bastante o mucho $(83,3 \%)$, llegan a aburrirse (66,7\%), en general, porque se utilizan un mismo juego $(50 \%)$ o porque juegan solos (37,5\%). El 100\% de los niños y niñas creen que han aprendido a través del programa de intervención educativa, y que tanto la actividad física $(91,7 \%)$, como la alimentación saludable $(90,9 \%)$ han cambiado sus hábitos de vida.
Por ello, creemos que sería interesante integrar este tipo de programas en el modelo educativo de los centros, al tratarse de un programa que educa y motiva al cambio de conducta desde edades tempranas utilizando las dinámicas propias de los juegos, que son tan cercanas a los niños y niñas.

También consideramos que es necesario que a corto plazo se plantee introducir en el curriculum educativo de los menores un espacio para la educación en hábitos de vida saludables. Y no que este tipo de educación quede al azar y al interés del docente de formar a sus alumnos y alumnas en este tema. El hecho de que la educación en hábitos de vida saludable forme parte del curriculum aseguraría a largo la adquisición de conductas saludables y la prevención de las enfermedades no transmisibles relacionadas con los malos hábitos, repercutiendo en una mejora de la salud para toda la sociedad.

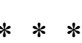

"El Proyecto de investigación PROVITAO Ref. OBE05 ha sido realizada con fondos para investigación de la Fundación CajaCanarias".

\section{REFERENCIAS}

AGENCIA ESPAÑOLA DE SEGURIDAD ALIMENTARIA (AECOSAN). Ministerio de Sanidad y Consumo. NAOs. Invertir la tendencia de la obesidad. Estrategia para la nutrición, actividad física y prevención de la obesidad. 2005. Disponible en: <http://www.aecosan.msssi.gob.es/AECOSAN/web/nutricion/seccion/estrategia_naos.shtml>. Acceso el: 30 jul. 2016.

ALFAGEME, B., SÁNCHEZ, P. Un instrumento para evaluar el uso y actitudes hacia los videojuegos. Píxel-Bit: Revista de medios e Educación, n. 20, p. 17-32, 2003.

ALVES, Lynn. Games e interatividade: mapeando possibilidades. Obra digital, n. 5, sep. 2013. Disponible en: $<$ http://revistesdigitals.uvic.cat/index.php/obradigital/article/view/28/32>. Acceso el: 30 jul. 2016.

BARANOWSKI, T. et al. Playing for real - video games and stories for health-related behavior change. American Journal of Preventive Medicine, v. 34, n. 1, p. 74-82, 2008.

BARROS, Marina. et al. The design in the development of exergames: a new game for the contribute to control childhood obesity. Design, user experience, and usability. Health, learning, playing, cultural, and cross-cultural user experience. Volume 8013 of the series Lecture Notes in Computer Science, p. 491-500, 2013. Disponible en: $<\mathrm{http}$ ///ink.springer.com/chapter/10.1007\%2F978-3-642-39241-2_54>. Acceso el: 30 jul. 2016.

BELTRÁN-CARRILLO, V. J.; VALENCIA-PERIS, A.; MOLINA-ALVENTOSA, J. P. Los videojuegos activos y la salud de los jóvenes: revisión de la investigación. Revista Internacional de Medicina y Ciencias de la Actividad Física y el Deporte, v. 10, n. 41, p. 203-219, 2011.

CLARK, R. A. et al. Validity and reliability of the Nintendo Wii Balance Board for assessment of standing balance. Gait Posture, v. 31, n. 3, p. 307-310, 2010.

CUBEROS, Ramón. et al. Exergames para la mejora de la salud en niños y niñas en edad escolar: Estudio a 
partir de hábitos sedentarios e índices de obesidad. 2015. Disponible en: $<$ http://relatec.unex.es/article/view/1471>. Acceso el: 30 jul. 2016.

CUSSÓ SEGURA, X.; GARRABOU SEGURA, R. La transición nutricional en la España contemporánea: las variaciones en el consumo de pan, patatas y legumbres (1850-2000). Investigaciones de Historia Económica. v. 3, n. 7, p. 69-100, 2007.

Disponible en: $<$ https://www.researchgate.net/profile/Carina_Gonzalez/publication/257867510_EMODIANA_Un instrumento_para_la_evaluacion_subjetiva_de_emociones_en_ninos_y_ninas/links/00b49525f $\mathrm{fc} 46 \mathrm{~b} 7 \mathrm{a} 185000000$. pdf $>$. Acceso el: 30 jul. 2016.

EZZATI, Majid; RIBOLI, Elio. Behavioral and dietary risk factors for noncommunicable diseases. The New England Journal of Medicine, n. 369, p. 954-964, 2013.

FALCADE, Ana. et al. Análise do consumo de oxigênio, da frequência cardíaca e equivalente metabólico obtidos através de um videogame ativo. Revista Inspirar. Movimiento \& Saúde, v. 5, n. 6, p. 20-24, nov./dic. 2013. Disponible en: $<$ http://inspirar.com.br/revista/?p=3033>. Acceso el: 30 jul. 2016.

FRENK, J. Abordando los desafíos de la salud mundial a través de la nutrición mejorada. World Health Organization. 2013. Disponible en: <http://www.who.int/pmnch/media/press/2012/20120619_articula_sobre_g20_jfrenk/ es/>. Acceso el: 30 jul. 2016.

GONZALEZ, C. S. et al. TANGO:H: Creating Active Educational Games for Hospitalized Children. Management Intelligent Systems, v. 220 of the series Advances in Intelligent Systems and Computing, p. 135-142. 2013. Disponible en: <http://link.springer.com/chapter/10.1007/978-3-319-00569-0_17>. Acceso el: 30 jul. 2016.

GONZÁLEZ, C. S. et al. Learning healthy lifestyles through active videogames, motor games and the gamification of educational activities. Computers in Human Behavior, v. 55, Part A, p. 529-551, Feb. 2016. Disponible en: $<$ http://www.sciencedirect.com/science/article/pii/S0747563215301266>. Acceso el: 30 jul. 2016.

GONZÁLEZ-GONZÁLEZ, C. S; BLANCO-IZQUIERDO, F. Designing social videogames for educational uses. Computers \& Education, v. 58, n. 1, p. 250-262, 2012. Disponible en: $<$ http://www.sciencedirect.com/science/ article/pii/S0360131511001928>. Acceso el: 30 jul. 2016.

GONZÁLEZ-GONZÁLEZ, C. S.; NAVARRO ADELANTADO, V. A structural theoretical framework based on motor play to categorize and analyze active video games. Games and Culture, v. 2, n. 7-8, p. 690-719, mar. 2015. Disponible en: $<$ https://www.researchgate.net/publication/272172015_A_Structural_Theoretical_Framework_Based_on_Motor_Play_to_Categorize_and_Analyze_Active_Video_Games $>$.Acceso el: 30 jul. 2016 .

GONZALEZ, C. S.; NAVARRO-ADELANTADO, V.; CAIROS, M. Un instrumento para la evaluación subjetiva de emociones en niños y niñas. In: CONGRESO INTERNACIONAL DE INTERACCIÓN PERSONA ORDENADOR, 14., Madrid, 2013. Actas electrónicas... Madrid, 2013. p. 69-74. Disponible en: $<$ http://aipo.es/files/actas/ ActasInteraccion2013.pdf>. Acceso el: 15 oct. 2016.

GUIXERES, J. et al. Terapias en obesidad infantil: estudio de los videojuegos activos como promotor del ejercicio físico. Alto Rendimiento, 2011. Disponible en: $<$ http://altorendimiento.com/terapias-en-obesidad-infantil-estudio-de-los-videojuegos-activos-comopromotor-del-ejercicio-fisico/>. Acceso el: 30 jul. 2016.

HILLIER, A. Childhood overweight and the built environment: making technology part of the solution rather than part of the problem. The ANNALS of the American Academy of Political and Social Science, v. 615, n. 1, p. 56-82, Jan. 2008.

INSTITUTO BRASILEIRO DE DEFESA DO CONSUMIDOR (IDEC). Revista do IDEC - Infância acima do peso, $\mathrm{n}^{\circ}$ 201, p. 14-17, ago. 2015. Disponible en: <http://www.idec.org.br/em-acao/revista/problemas-de-peso/ materia/infancia-acima-do-peso>. Acceso el: 30 jul. 2016.

LÓPEZ, J. et al. The video game as practice for developing virtual reality sports jumping skills in children 5 years. Case study of innovative practices in educational institutions of Bogotá, Colombia. In: New developments in pure and applied mathematics. Proceedings of the International Conference on Pure Mathematics -Applied Mathematics (PM-AM 2015).Vienna, Austria. March 15-17, 2015. Disponible en: <http://www.inase.org/library/2015/vienna/ bypaper/MAPUR/MAPUR-34.pdf>. Acceso el: 30 jul. 2016.

MOYA MARTÍNEZ, P. Coste-efectividad de un programa de actividad física de tiempo libre para prevenir el sobrepeso y la obesidad en niños de 9-10 años. Gaceta Sanitaria. v. 25, n. 3, p. 198-204, 2011. 
ORGANIZACIÓN MUNDIALDE LA SALUD (OMS). Estrategia mundial sobre régimen alimentario, actividad y salud de la 57 ${ }^{a}$ Asamblea Mundial de la Salud. Resolución WHO_57.17. 2004. Disponible en: <http://www. who.int/dietphysicalactivity/strategy/eb11344/strategy_spanish_web.pdf >. Acceso el: 30 jul. 2016.

Obesidad y sobrepeso. 2014. Disponible en: <http://www.who.int/mediacentre/factsheets/fs311/es/>. Acceso el: 30 jul. 2016.

PROGRAMA DE VIDEOJUEGOS ACTIVOS PARA EL TRATAMIENTO AMBULATORIO DE LA OBESIDAD (PROVITAO). El proyecto. 2016. Disponible en: <http://provitao.ull.es/web/index.html $>$. Acceso el: 30 jul. 2016.

SOCIEDAD ESPAÑOLA PARA EL ESTUDIO DE LA OBESIDAD (SEEDO). La obesidad ahora si una enfermedad de peso. 2013. Disponible en: $<$ http://www.seedo.es/index.php/la-obesidad-ahora-si-una-enfermedad-de-peso $>$. Acceso el: 30 jul. 2016.

SERRANO, E. Videojuegos para la Salud. In: CURSO DE COMUNICACIÓN Y SALUD. Nuevos escenarios y tendencias en tiempos de crisis, 6., Madrid, 2010. Anais... Madrid: Facultad de Ciencias de la Información de la UCM, 2010. Disponible en: <http://eprints.sim.ucm.es/13410/1/comunicacionysalud.pdf>. Acceso el: 30 jul. 2016.

SOUTHHALL J. E.; OKELY, A. D.; STEELE, J. Actual and perceived physical competence in overweight and nonoverweight children. Pediatric Exercise Science, n. 16, p. 15-24, 2004.

ZURANO CONCHES L. Investigadores estudian la eficacia en la población infantil de los videojuegos activos como facilitadores del ejercicio físico. Nota de Prensa. Universitat Politécnica de Valencia, 2011.

Recebido em:02/06/2016

Aprovado em: 01/10/2016 